\title{
Getting that inner glow in non-transgenic animals
}

\author{
Yevtodiyenko, A. et al. Nat Commun 12, 2680 (2021)
}

Bioluminescent tools can give different animals an inner glow that researchers can then take advantage of to image what's going on inside of their subjects. Bioluminescence imaging (BLI) lacks some specificity that other imaging modalities offer, but the imaging itself is relatively noninvasive and offers longitudinal monitoring of different biological processes. Getting the necessary components in to the animals can however be a bit more involved. The product of a biochemical reaction, bioluminescence comes from light generated when the enzyme luciferase oxidizes its substrate, luciferin. Luciferase and luciferin (as well as many related variants) can be found in a number of organisms - perhaps most notably, the firefly - but lab mice, and most other animals for that matter, don't come by the molecules naturally.

Researchers that want to use bioluminescence imaging (BLI) must generate transgenic animals that express luciferin in cells or tissues of interest, after which they can be injected with luciferase to prompt the light-generating chemical reaction. The process works well and has yielded valuable insights in many disease areas, but creating transgenic models takes time, money, and extra animals. It's also limited to those species that can be genetically modified in the first place. The instruments involved can also be expensive and cumbersome - most BLI systems require a light-tight box and a cooled charge-coupled device camera; these can be constrained by subject size and are not always suitable for larger, non-rodent species. Such animals, including primates, canines, cats, and rabbits, are however important in research - particularly for toxicology and drug testing before candidates can be moved on to clinical trials.

Elena Goun and her lab, now at the University of Missouri, Columbia, have been working to advance BLI technologies, with a motivation to help improve the quality of animal experiments while reducing the numbers needed. Writing in Nature Communications, she and colleagues present a low cost ( $\sim 00$ to 600 USD), portable BLI method that's compatible with non-transgenic animals.

The portable bioluminescent system (PBL) relies on injections, rather than genetic modification. First, the subject is intraperitoneally injected with a caged

Portable bioluminescence system (PBL)

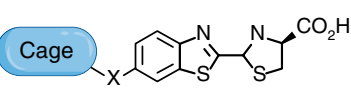

Caged luciferin probe

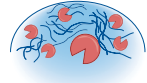

Luciferase plug

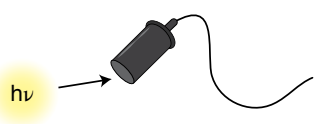

Light detector

The three components of the PBL. Credit: Yevtodiyenko, A. et al. (Springer Nature) 2021

probe - in this case, luciferin that has been rendered inert until the chemical bonds 'caging' it are broken by specific biological processes or enzymes in the body. Next, the animal is subcutaneously injected with a small plug that contains the luciferase enzyme. As the luciferin is uncaged, it will flow through the blood stream and react with the luciferase as it passes by. A portable light device developed by Goun and a team of international collaborators can then be held above where the plug was injected, where it will record any bioluminescence. The stronger the light signal, the greater the reaction in the animal.

Following validation of the components in vivo, the team tested the PBL's ability to detect an intracellular enzyme, cytochrome P450 (CYP450). CYP450 is a liver enzyme involved in drug metabolism; testing for its activation is an important part of assessing the toxicity of therapeutic candidates. This is however a highly invasive process that can involve many animals that must undergo multiple blood draws before being sacrificed for final analysis. The most common isoform of CYP450, cytochrome P450 3A (Cyp3a) can be activated with injections of dexamethasone (DEX), so the team injected DEX into cohorts of luciferase-expressing and non-transgenic, wild-type FVB mice and then recorded for enzymatic activity under a conventional BLI camera and the smaller PBL device. They observed comparable readouts. They then compared their imaging results with conventional measures of Cyp3a activity, which involved sacrificing animals to analyze expression in their livers. PBL proved up to par.

Moving on from mice, the team tried the PBL on a larger animal: dogs. First, they confirmed the system was indeed safe for use in canines. Though luciferin is extensively in mice without adverse effects, data wasn't available for dogs. With no clinical signs of toxicology observed in the dogs' blood serum, the team could then determine if the amount of bioluminescence from the luciferase corresponded to luciferin concentrations. It did - all while requiring 10x less luciferin than that needed in mice to see the same signal, says Goun. “This means much lower doses of 'caged' luciferin probes are needed, making production of the compounds more practical," she says.

In a final proof of principle, the team injected a luciferase plug and three doses of luciferin into the arm of a human cadaver donated to the Swiss Federal Institute of Technology Lausanne; the light detector picked up light for fifteen minutes, with a signal proportional to luciferin concentration.

The PBL system was years in the making - Goun says she'd been dreaming of developing it since 2011, but it took time to find the optical engineers, including the National Institute of Standard and Technology's Sergey Polyakov and George Eppeldauer, to collaborate with on the necessary components. From this first prototype device, she hopes to go wireless with implantable sensors, similar to those used to record glucose levels. The team is also exploring other kinds of caged luciferins.

Meanwhile, Goun hopes the technology will prove useful for those labs that cannot afford the expensive equipment currently required for BLI. Avoiding the need for transgenic animals - including rodents should also help reduce the total number of animals needed for a BLI study, as will the ability to measure drug toxicity without needing to sacrifice test subjects.

\section{Ellen Neff}

Published online: 14 June 2021

https://doi.org/10.1038/s41684-021-00798-9 